\title{
BMJ Open Using structural and functional MRI as a neuroimaging technique to investigate chronic fatigue syndrome/myalgic encephalopathy: a systematic review
}

\author{
Basim Almutairi, ${ }^{1,2}$ Christelle Langley, ${ }^{3}$ Esther Crawley, ${ }^{4}$ Ngoc Jade Thai ${ }^{1}$
}

To cite: Almutairi B, Langley C, Crawley $\mathrm{E}$, et al. Using structural and functional MRI as a neuroimaging technique to investigate chronic fatigue syndrome/ myalgic encephalopathy: a systematic review. BMJ Open 2020;10:e031672. doi:10.1136/ bmjopen-2019-031672

- Prepublication history and additional material for this paper are available online. To view these files, please visit the journal online (http://dx.doi org/10.1136/bmjopen-2019031672).

Received 16 May 2019 Revised 13 March 2020 Accepted 11 May 2020

\section{Check for updates}

(c) Author(s) (or their employer(s)) 2020. Re-use permitted under CC BY-NC. No commercial re-use. See rights and permissions. Published by BMJ.

${ }^{1}$ Clinical Research and Imaging Centre, Bristol Medical School, Faculty of Health Sciences, University of Bristol, Bristol, UK ${ }^{2}$ Radiology \& Imaging Centre, King Saud Medical City, Saudi Ministry of Health, Riyadh, Saudi Arabia

${ }^{3}$ The Herchel Smith Building for Brain and Mind Sciences, Department of Psychiatry, Cambridge University,

Cambridge, UK

${ }^{4}$ Centre of Child and Adolescent Health, Bristol Medical School, Faculty of Health Sciences, University of Bristol, Bristol, UK

Correspondence to

Dr Ngoc Jade Thai;

jade.thai@bristol.ac.uk

\section{ABSTRACT}

Objective This systematic review aims to synthesise and evaluate structural MRI (sMRI) and functional MRI (fMRI) studies in chronic fatigue syndrome/myalgic encephalomyelitis (CFS/ME).

Methods We systematically searched Medline and Ovid and included articles from 1991 (date of Oxford diagnostic criteria for CFS/ME) to first April 2019. Studies were selected by predefined inclusion and exclusion criteria. Two reviewers independently reviewed the titles and abstracts to determine articles for inclusion, full text and quality assessment for risk of bias.

Results SMRI studies report differences in CFS/ME brain anatomy in grey and white matter volume, ventricular enlargement and hyperintensities. Three studies report no neuroanatomical differences between CFS/ME and healthy controls. Task-based fMRI investigated working memory, attention, reward and motivation, sensory information processing and emotional conflict. The most consistent finding was CFS/ME exhibited increased activations and recruited additional brain regions. Tasks with increasing load or complexity produced decreased activation in taskspecific brain regions.

Conclusions There were insufficient data to define a unique neural profile or biomarker of CFS/ME. This may be due to inconsistencies in finding neuroanatomical differences in CFS/ME and the variety of different tasks employed by fMRI studies. But there are also limitations with neuroimaging. All brain region specific volumetric differences in CFS/ME were derived from voxel-based statistics that are biased towards group differences that are highly localised in space. fMRI studies demonstrated both increases and decreases in activation patterns in CFS/ME, this may be related to task demand. However, fMRI signal cannot differentiate between neural excitation and inhibition or function-specific neural processing. Many studies have small sample sizes and did not control for the heterogeneity of this clinical population. We suggest that with robust study design, subgrouping and larger sample sizes, future neuroimaging studies could potentially lead to a breakthrough in our understanding of the disease.

\section{INTRODUCTION}

Chronic fatigue syndrome (CFS), also known as myalgic encephalomyelitis/ myalgic encephalopathy (ME) is a disorder
Strengths and limitations of this study

To the best of our knowledge, this is the first systematic review of neuroimaging studies that have investigated chronic fatigue syndrome/Myalgic Encephalomyelitis/myalgic encephalomyelitis (CFS/ ME) using MRI.

- We reviewed both structural MRI and functional MRI (fMRI) studies of CFS/ME.

- We identified common limitations across the neuroimaging studies and make recommendations for future research.

- We were unable to find conclusive evidence for neural biomarkers of CFS/ME.

- The main limitation of the current systematic review is that a meta-analysis was not possible because of the different methodologies across the studies, such as fMRI studies using a variety of tasks to assess different cognitive functions.

characterised by persistent fatigue which lasts for at least 4 months or 6 months (depending on the diagnostic criteria) and is associated with a variety of symptoms. One of the most common symptoms is cognitive dysfunction which is reported in $>94 \%$ of adults. ${ }^{1} \mathrm{CFS} / \mathrm{ME}$ is relatively common. A recent meta-analysis performed by Johnston et al (2014) indicates a prevalence of $0.76 \%$ (95\% CI $0.23 \%$ to $1.29 \%$ ) based on clinically confirmed cases in several countries. ${ }^{2}$ Over $50 \%$ of adults who access specialist care are unemployed because of CFS/ME. ${ }^{3}$ The aetiology and pathophysiology of CFS/ME are not known and the underlying mechanism for cognitive dysfunction is not understood.

Imaging techniques such as MRI have been used to aid clinical diagnoses for decades. A variety of MRI techniques have been used in neurology from structural MRI (sMRI) in lesion detection to functional MRI (fMRI) applications for neurosurgical planning. Recently MRI has been used to examine fatigue and cognition in CFS/ME. This 
systematic review aims to evaluate the use of sMRI and fMRI to investigate CFS/ME. We also aim to provide an insight into what MRI can offer to our understanding of cognitive dysfunction in CFS/ME. Finally, we will make suggestions for future directions of research.

\section{MATERIAL AND METHODS}

We searched Medline and Ovid and included articles from 1991 (Oxford diagnostic criteria for CFS/ME) to April 2019. We included all English language studies using MRI to investigate CFS. We used the following key words (and abbreviations) for CFS/ME: 'chronic fatigue syndrome', 'fatigue syndrome, chronic', 'myalgic encephalomyelitis', 'myalgic encephalopathy', 'CFS', 'ME' or 'CFS/ME'. To detect all structural and functional studies which used MRI in participants with CFS/ME, we used the following keywords for imaging techniques: 'MRI', 'MRI', 'structural MRI', 'sMRI', 'functional MRI', 'functional MRI', 'fMRI', 'resting state functional MRI', 'resting-state functional MRI', 'resting-state functional MRI', 'resting state functional MRI', 'rsfMRI' and 'rs-fMRI'. The full search strategy is provided in (online supplementary file 1) 'SearchFile MEDLINE and OVID'.

We included all studies which used one of the five major CFS/ME definition criteria; Fukuda, Centers for Disease Control and Prevention(CDC), National Institute for Health and Care Excellence, Canadian or Oxford criteria and there was no age restriction. Duplicates, case study articles and editorials were excluded. Two reviewers (BA, CL) independently reviewed the titles and abstracts of identified studies and potentially relevant articles were identified for full-text review. Two reviewers independently reviewed full-text articles to determine which articles were eligible. Disagreements were resolved by discussion until consensus was reached.

\section{Risk of bias assessment}

We assessed study quality and risk of bias using the following criteria outlined in Nichols $e t a:^{4}$ clearly stated research objective; recruitment procedure; inclusion/ exclusion criteria; description of sample demographics; reporting of imaging methodology; and whether comparison groups were used. These criteria have been set with the aim to increase the reproducibility of research for neuroimaging studies using MRI. Stating clearly the recruitment procedure, inclusion/exclusion criteria, population demographics and comparison group enables a critical reader to evaluate the study and to determine whether the sample may be susceptible to bias and whether the results are generalisable. ${ }^{4}$ If a study fully reported all criteria, it was considered a high-quality study with low risk of bias. If it failed to report one criterion, it was considered a medium quality paper. Finally, if it failed to report two or more criteria, it was considered a low-quality study with a high risk of bias. Given the low number of identified studies, we did not exclude any studies based on quality assessment, but we reviewed the

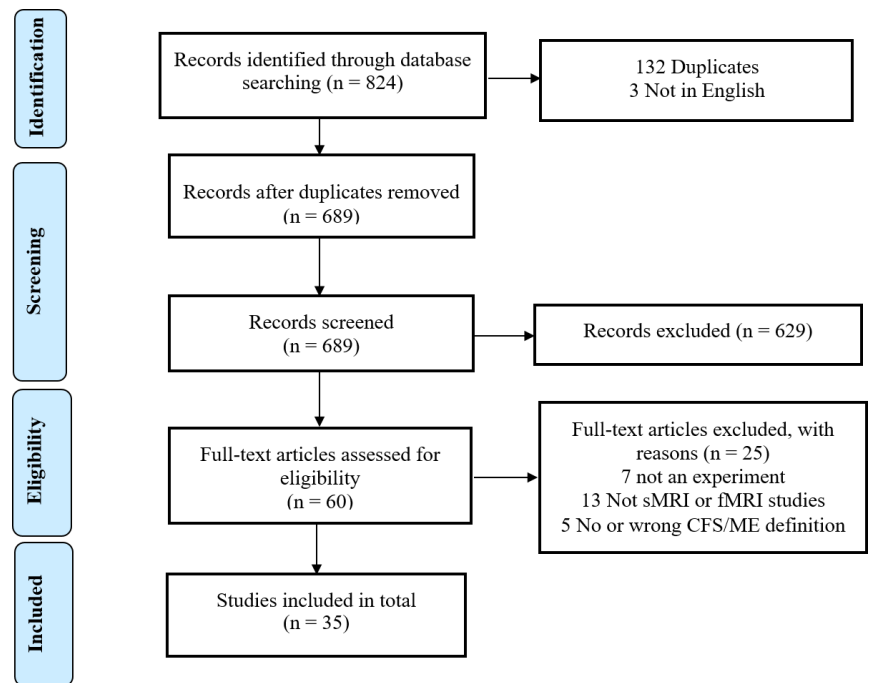

Figure 1 Preferred Reporting Items for Systematic Reviews and Meta-Analyses (PRISMA) flow chart showing the method followed. The PRISMA flow diagram for the systematic review detailing the database searches, the number of abstracts screened and the full texts retrieved. CFS/ME, chronic fatigue syndrome//myalgic encephalomyelitis; fMRI, functional MRI; sMRI, structural MRI.

results taking study quality into consideration. We did not submit this work to an ethics committee because it is a systematic review of the literature.

\section{Patient and public involvement}

No patient or public were involved.

\section{RESULTS}

A total of 824 papers was identified. Of these, 132 were duplicates and 3 were not in English (see figure 1). Of the remaining 689, there were 629 that did not fit our eligibility criteria leaving 60 papers for full-text review. Of these, 20 studies were excluded because they did not use sMRI or fMRI applications. A further five were excluded because the CFS/ME diagnostic criteria used were not clear. Therefore, we extracted data from 35 studies. Of the papers included, 19 were sMRI and 16 were fMRI studies.

\section{CFS/ME diagnostic criteria and MRI images acquisition}

Details on the diagnostic criteria as well as MRI images acquisition are available in table 1 for sMRI studies and table 2 for fMRI studies.

\section{Image analysis}

\section{- sMRI}

In 13 studies, quantitative computational analysis, such as statistical parametrical mapping (SPM) and FMRIB Software Library (FSL), of images was carried out by utilisation of an automated technique. ${ }^{5-16}$ However, six studies in sMRI relied on visual inspection by two radiologists and where there was disagreement a third radiologist was involved. ${ }^{17-22}$ 
Table 1 Summary of 19 sMRI studies in CFS/ME

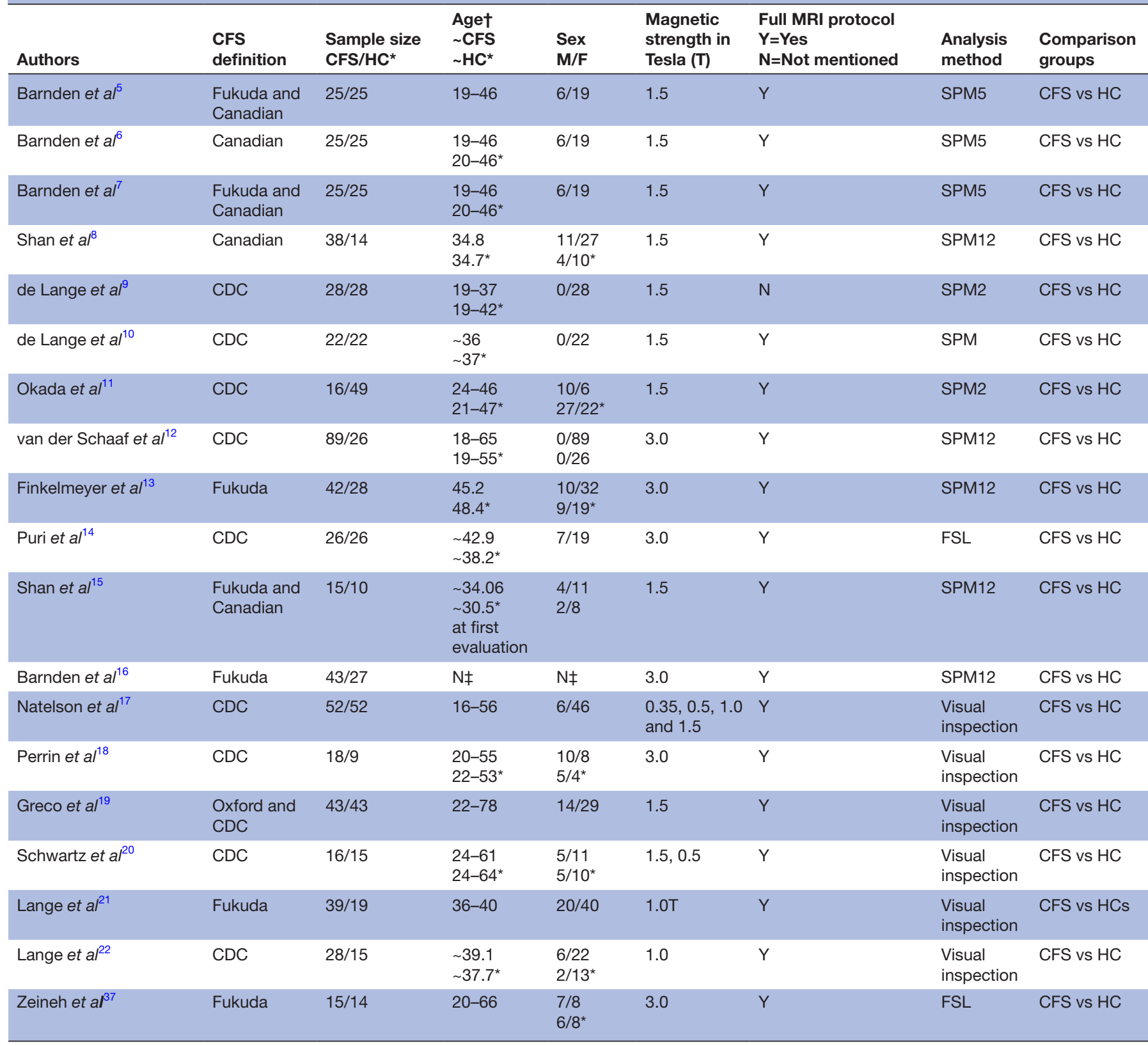

*Healthy controls

†Some studies provided average age and others provided a range

$\ddagger$ Not mentioned

CDC, Centers for Disease Control and Prevention; CFS, chronic fatigue syndrome; F, female; FSL, FMRIB Software Library; HC, healthy controls; M, male; ME, myalgic encephalomyelitis; sMRI, structural MRI; SPM, statistical parametric mapping.

\section{fMRI}

All of the 16 studies used quantitative computational analysis, such as SPM, ${ }^{823-32}$ Analysis of Functional NeuroImages software, Individual Brain Activation Maps (XBAM) software s3 $^{34}$ and FSL. ${ }^{35}$

\section{SMRI results}

Details of the 19 sMRI studies are available in table 1.

General findings

Of the 19 studies included in this systematic review, 16 showed some structural differences between CFS/ME and healthy controls. These included both grey matter volume and white matter volume reduction, ventricular enlargement, white matter hyperintensities, lesions and cortical thickening. In contrast, three studies did not reveal any differences between participants with CFS/ME and healthy controls and therefore questioned the ability of sMRI scans to detect brain changes in CFS/ME. ${ }^{6} 1218$

Radiological reporting

To evaluate and compare the sMRI of CFS/ME and healthy controls these studies used one reviewer, ${ }^{19}$ two neuroradiologists ${ }^{17182122}$ or three neuroradiologists ${ }^{20}$ 
Table 2 Summary of $16 \mathrm{fMRI}$ studies in CFS/ME

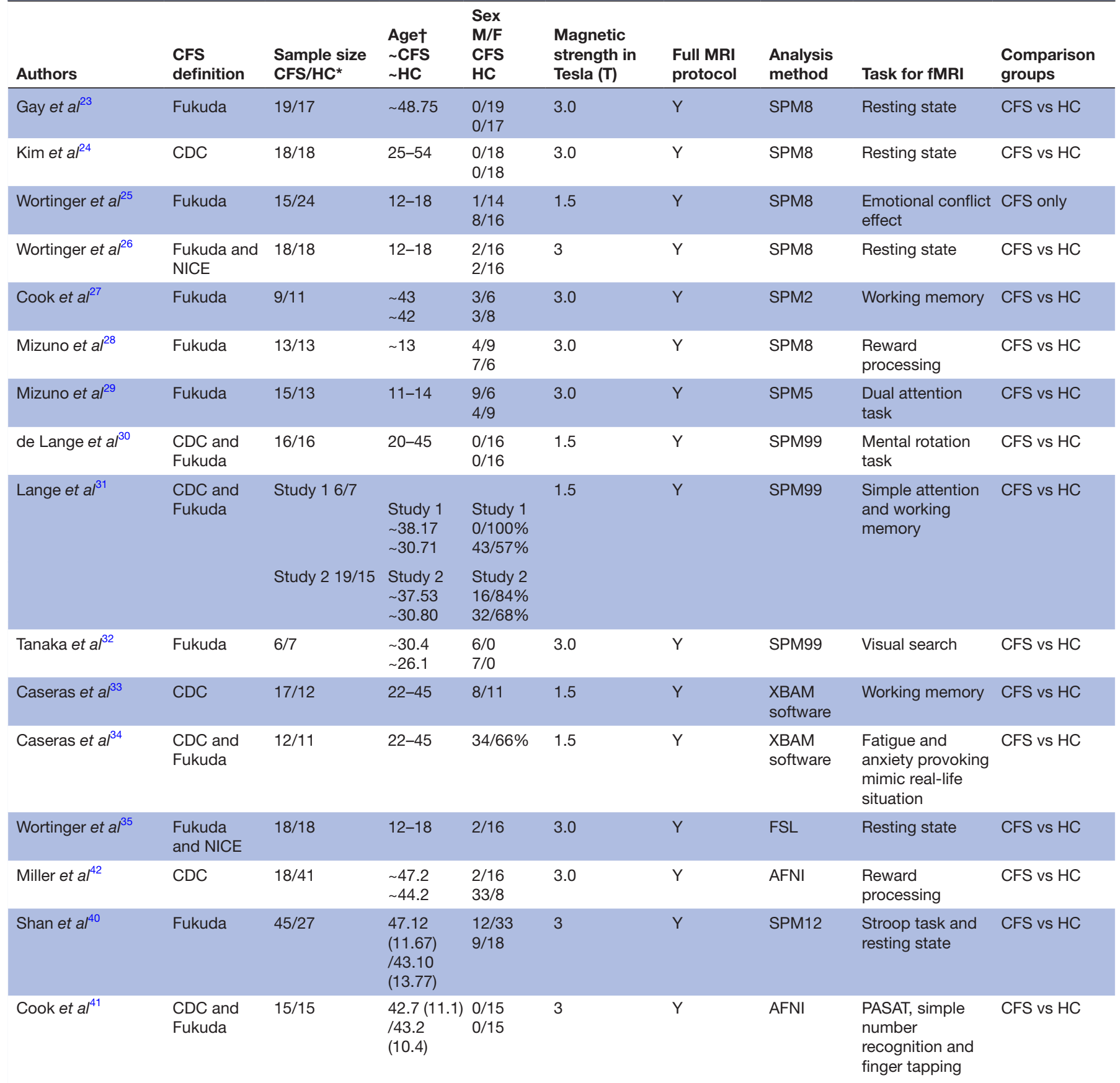

*Healthy controls

†Some studies provided average age and others provided a range

AFNI, Analysis of Functional Neurolmages; CDC, Centers for Disease Control and Prevention; CFS, chronic fatigue syndrome; F, female ; fMRI, functional MRI; FSL, FMRIB Software Library; HC, healthy control; M, male; ME, myalgic encephalomyelitis; NICE, National Institute for Health and Care Excellence; PASAT, Paced Auditory Serial Addition Test; SPM, statistical parametrical mapping; XBAM, Individual Brain Activation Maps.

to visually inspect the images. Two MRI studies found ventricular enlargement, ${ }^{1722}$ while three studies reported white matter hyperintensities or abnormalities, which were defined as lesions, identified by high signal intensity on T2 or proton density-weighted pulse sequences. ${ }^{17} 2021$ In these studies, age was only accounted for by using agematched healthy controls. Lange $e t a l^{21}$ reported that $41 \%$ of the MRI scans showed abnormalities. Further changes or lesions were reported in the supratentorial periventricular white matter, $^{19}$ periventricular white matter, subcortical white matter and in the centrum semiovale. ${ }^{20}$ However, a longitudinal study, with 1 year follow-up, failed to detect any differences between CFS/ $\mathrm{ME}$ and healthy control groups at baseline and after a year in cerebrospinal fluid, white matter hyperintensities, ventricular volume and failed to observe any abnormalities in the CFS/ME group. ${ }^{18}$ These studies used manual segmentation of the cerebrospinal fluid. The resultant 
images were registered into a standard space, segmented into 12 segments, defined by the mid-sagittal plane. Images were manually checked to exclude irrelevant areas, and finally, checked by an experienced neuroradiologist using the Scheltens et al method. ${ }^{18} 36$

White matter volume reduction was reported in five studies $^{813-1537}$ and white matter changes or lesions were reported in three further studies. ${ }^{719} 20$ In a recent study, Finkelmeyer $e t a l^{13}$ showed a substantial increase in grey matter volume and decrease in white matter volume in CFS/ ME compared with healthy controls. ${ }^{13}$ Moreover, they used an automated voxel-wise analysis, Computational Anatomy Toolbox (CAT12) in SPM12, which showed that the insula and amygdala had increased grey matter volume in the CFS/ ME group, while the midbrain, pons and right temporal lobe, had decreased white matter volume. ${ }^{13}$ Two studies, by the same research group, showed a marked reduction in the white matter volume in left inferior front-occipital fasciculus in participants with CFS/ME compared with healthy controls. ${ }^{8}{ }^{15}$ Puri $e t a l^{4}$ found a reduction in white matter volume in the left occipital lobe as well as the posterior part of the left parahippocampal gyrus in the CFS/ME group compared with the healthy control group. ${ }^{14}$ Changes in white matter observed on T2-weighted images in the right middle temporal lobe were related to cognition. Authors were able to demonstrate that white matter volume was negatively correlated with CFS/ME disease duration. ${ }^{57}$ This means that white matter volumes decreased with increased disease duration. ${ }^{57}$ Zeineh et $a l^{37}$ showed bilateral white matter atrophy in supratentorial was present in CFS/ME. ${ }^{37}$

Grey matter volume reduction was the main result in five studies. ${ }^{5-1114}$ There was reduced global grey matter volume in three studies ${ }^{5910}$ and regional grey matter volume difference in the two studies. ${ }^{11}{ }^{14}$ The reduction in grey matter volume was observed in the occipital lobes, right angular gyrus, left parahippocampal gyrus and in the bilateral prefrontal cortex. ${ }^{1114}$ Grey matter volume reduction has been associated with functional deficits, that may be influenced by pain $^{38} 39$ illness or age factors, thereby having a detrimental impact on quality of life for participants with CFS/ME. ${ }^{114}$

\section{Longitudinal studies}

Three studies compared sMRI in participants with CFS/ME across two time points. ${ }^{1015} 18$ de Lange $e a^{10}$ used MRI to look at the effects of cognitive behavioural therapy (CBT) on brain volume. At baseline, they described a decrease in grey matter volume in participants with CFS/ME compared with healthy controls. They also described an increase in grey matter volume between pretreatment and post-treatment MRI in the lateral prefrontal cortex in participants with CFS/ $\mathrm{ME}$, but this region remained unchanged in healthy controls. The increased grey matter volume in the lateral prefrontal cortex was correlated with health status, processing speed and physical activity. ${ }^{10}$ Shan $e t a l^{15}$ compared MRI images of participants with CFS/ME and healthy controls acquired 6 years apart. They found a substantial decrease in white matter volume in the left inferior fronto-occipital fasciculus in the CFS/ME group compared with the healthy control group.
Perrin $e \mathrm{al}^{18}$ conducted a 1-year follow-up and demonstrated no significant abnormalities or differences between baseline and the 12-month follow-up MRI in CFS/ME compared with controls. $^{18}$

\section{fMRI results}

Sixteen articles that employed fMRI were identified. Table 2 is a summary of these studies.

\section{General findings}

Sixteen fMRI studies were identified in this systematic review, 5 employed resting state fMRI (rs-fMRI) and 11 used multiple tasks to investigate cognitive functioning in CFS/ME.

\section{rs-fMRI and functional connectivity}

Four out of the five rs-fMRI studies reported decreased functional connectivity in participants with CFS/ME compared with healthy controls. ${ }^{23263540}$ Two studies reported a decrease in functional connectivity between the salience network and the right posterior insula. ${ }^{26}{ }^{35}$ Wortinger $e t a l^{35}$ reported a decrease in functional connectivity between the salience network and the right middle, posterior and anterior insula as well as between the salience network and superior temporal gyrus, precentral gyrus and thalamus, which are brain regions outside of the classic boundaries of the salience network. ${ }^{35}$ Wortinger et al showed a reduction in functional connectivity between the right dorsal anterior insula and the right posterior parietal cortex of the central executive network. ${ }^{26}$ Gay $e t a l^{23}$ found a disruption in the intrinsic connectivity within the left frontoparietal network. More specifically, they found reduced coupling of activity between the left superior frontal gyrus and the five networks they investigated. These five networks included the default mode network, salience network, sensory motor network, and the left and right frontoparietal networks. Also, they found a decrease in functional connectivity between the salience network and the left posterior cingulate cortex. The sensory motor network showed decreased functional connectivity with the left anterior mid-cingulate cortex. ${ }^{23}$ Shan $e t a l^{t 0}$ found decreased functional connectivity between the medial prefrontal cortex and both inferior parietal lobules. ${ }^{40}$ On the other hand, Kim $e t ~ a l ~{ }^{24}$ reported an increase in functional connectivity between the posterior parietal cortex and the dorsal anterior cingulate cortex, rostral anterior cingulate cortex, middle temporal cortex and precuneus in participants with CFS/ ME compared with healthy controls. ${ }^{24}$ Collectively the findings from rs-fMRI studies suggest dysfunctional connectivity across a number of neural networks in CFS/ME.

\section{fMRI and cognition}

\section{- Memory}

Working memory was investigated in CFS/ME groups using a variety of tasks. Cook $e$ t al in a recent study (2017) and an older study (2007) reported no differences between CFS/ME and healthy controls in simple non-fatiguing tasks like finger tapping or auditory monitoring. ${ }^{27}{ }^{41}$ However, CFS/ME participants showed significantly widespread increased cortical and subcortical activation throughout the complex and fatiguing cognitive task. ${ }^{27} 41$ Caseras $e t$ 
al showed that, during performance of the n-back task, participants with CFS/ME exhibited increased activation compared with healthy controls in medial prefrontal regions, during the 1-back condition. ${ }^{33}$ Conversely, in the more challenging conditions (2-back and 3-back conditions) participants with CFS/ME showed decreased activation in dorsolateral prefrontal and parietal cortices, which are working memory related brain regions. ${ }^{33}$ Moreover, they found that the CFS/ME group activated a large cluster in the right inferior/medial temporal cortex while performing 2-back and 3-back conditions not activated in healthy controls. ${ }^{33}$ Trend analysis of the load of the task showed statistically significant differences in activation in the brain between CFS/ME and the healthy control groups as task demand increased. ${ }^{33}$

\section{- Attention}

Mizuno et al reported that a dual attention task revealed activation in the left dorsal inferior frontal gyrus was greater in the dual task condition than in the two single task conditions in both healthy controls and adolescents with CFS/ME. In healthy controls, the level of activation was positively associated with the fatigue score and negatively correlated for the accuracy for story comprehension. In adolescents with CFS/ME, the activation of the dorsal anterior cingulate cortex and left middle frontal gyrus was only observed in the dual task condition. In addition, the levels of activation of the dorsal anterior cingulate cortex and left middle frontal gyrus were positively associated with both motivation and fatigue scores. ${ }^{29}$ In a recent study, Shan et al investigated CFS/ME using the Stroop task and could not find any differences between the groups. ${ }^{40}$ However, when they examined the default mode network they found lower functional connectivity between medial prefrontal cortex, left inferior parietal lobule, medial prefrontal cortex and posterior cingulate cortex in CFS/ME. ${ }^{40}$

\section{- Reward and motivation}

During a reward processing task/gambling task, the CFS/ME group showed significantly reduced activation in the right caudate and right globus pallidus compared with controls. Moreover, the decreased activation in the right globus pallidus was significantly associated with the elevation in mental fatigue and general fatigue, as evaluated by the multidimensional fatigue inventory. ${ }^{42}$ Another study using a different gambling task showed activation of the bilateral caudate, putamen, and thalamus in both the healthy control group and the adolescents with CFS/ME group, when using high monetary reward condition. ${ }^{28}$ In the low monetary reward condition activation of the bilateral caudate and thalamus was observed in both the healthy controls group and the adolescents CFS/ME group, but activation of the bilateral putamen was only observed in the healthy controls group. ${ }^{28}$

- Sensory information processing tasks

A mental rotation task showed that participants with $\mathrm{CFS} / \mathrm{ME}$ had stronger responses in visual structures. During error trials, the dorsal anterior cingulate cortex was activated in both groups. However, the ventral anterior cingulate cortex was activated only when healthy controls made an error and remained inactive when participants with CFS/ME made an error. ${ }^{30}$ During the Paced Auditory Serial Addition Test (PASAT) task, participants with CFS/ME demonstrated a significant increase in blood oxygen level dependant (BOLD) signal in bilateral premotor and left superior parietal regions. ${ }^{3041}$

A visual search task was used by Tanaka $e t a l^{32}$ to examine the task-dependent brain regions. They showed a reduction in activation in bilateral visual cortices, left superior and inferior parietal lobules, and left precentral gyrus, for the fatigue-inducing task in both participants with CFS/ME and healthy controls. Furthermore, the amount of decrease in activation was the same in both groups. Conversely, the activation of auditory cortices throughout the fatigue-inducing period did not change in the healthy controls but was reduced in the participants with CFS/ME. The amount of reduction was associated with fatigue which was measured immediately before the MRI session using a fatigue Visual Analogue Scale. ${ }^{32}$

When fatigue and anxiety were induced by mimicking real-life situations, the CFS/ME group reported fatigue and anxiety and exhibited an increase in activation in the occipitoparietal cortex, posterior cingulate gyrus and parahippocampal gyrus, as well as a reduction in functional connectivity between dorsolateral and dorsomedial prefrontal cortices compared with healthy controls. These results suggest a relationship between provocation of fatigue and activations in these brain areas. ${ }^{34}$

\section{- Emotional conflict}

Emotional conflict tasks indicated that the CFS/ME group were less able to engage the left amygdala and left mid-posterior insula in response to conflict than the healthy group. ${ }^{25}$ Moreover, there was an association between accuracy interference and conflict-related reactivity in the amygdala in adolescents with CFS/ ME. A significant decrease was observed in the left amygdala of adolescents with CFS/ME when compared with healthy controls. No difference was measured in the right amygdala between the groups. A significant decrease in the activity of the left mid-posterior insula was observed. ${ }^{25}$ No group differences between the two groups were reported in the right fronto-insular cortex, a key region of salience network responsible for integrating other salience network regions in the processing of emotional information, ${ }^{43}$ and dorsal anterior cingulate cortex. ${ }^{25}$

\section{QUALITY ASSESSMENT FOR RISK OF BIAS}

We applied the criteria from Nichols $e t$ al to assess study quality for risk of bias. ${ }^{4}$ See tables 3 and 4 for risk of bias assessment. The sMRI studies risk of bias assessment showed that study quality was highly variable. All fMRI studies were assessed to have low risk of bias and therefore considered high quality. 
Table 3 Shows risk of bias assessment for structural MRI studies

\begin{tabular}{|c|c|c|c|c|c|c|c|c|}
\hline Authors & $\begin{array}{l}\text { Research } \\
\text { objectives }\end{array}$ & $\begin{array}{l}\text { Recruitment } \\
\text { procedure }\end{array}$ & $\begin{array}{l}\text { Inclusion/ } \\
\text { exclusion }\end{array}$ & $\begin{array}{l}\text { Population } \\
\text { demographics }\end{array}$ & $\begin{array}{l}\text { Imaging } \\
\text { protocol }\end{array}$ & $\begin{array}{l}\text { Comparison } \\
\text { group }\end{array}$ & $\begin{array}{l}\text { Quantitative/ } \\
\text { narrative }\end{array}$ & $\begin{array}{l}\text { Risk of } \\
\text { bias }\end{array}$ \\
\hline Barnden et $a l^{5}$ & $Y$ & $\mathrm{Y}$ & $Y$ & Y & $\mathrm{Y}$ & CFS vs HC & $Q$ & Low \\
\hline Barnden et $a l^{6}$ & $\mathrm{Y}$ & $\mathrm{Y}$ & $\mathrm{Y}$ & Y & Y & CFS vs $\mathrm{HC}$ & $Q$ & Low \\
\hline Barnden et $a l^{7}$ & $\mathrm{Y}$ & $\mathrm{Y}$ & $\mathrm{Y}$ & $\mathrm{Y}$ & $\mathrm{Y}$ & CFS vs $\mathrm{HC}$ & Q & Low \\
\hline Shan et $a l^{8}$ & $\mathrm{Y}$ & $Y$ & Y & Y & Y & CFS vs $\mathrm{HC}$ & $Q$ & Low \\
\hline de Lange et $a l^{9}$ & Y & Y & Y & Y & $\mathrm{N}$ & CFS vs $\mathrm{HC}$ & Q & Medium \\
\hline Okada et al ${ }^{11}$ & Y & Y & Y & Y & $\mathrm{N}$ & CFS vs $\mathrm{HC}$ & Q & Medium \\
\hline $\begin{array}{l}\text { van der Schaaf } \\
\text { et } a l^{12}\end{array}$ & $\mathrm{Y}$ & $\mathrm{Y}$ & $\mathrm{Y}$ & $\mathrm{Y}$ & $\mathrm{Y}$ & CFS vs HC & Q & Low \\
\hline Finkelmeyer et $\left.\mathrm{a}\right|^{13}$ & Y & Y & Y & Y & Y & CFS vs $\mathrm{HC}$ & Q & Low \\
\hline Puri et $a l^{14}$ & $\mathrm{Y}$ & $\mathrm{N}$ & $\mathrm{Y}$ & Y & $\mathrm{Y}$ & CFS vs $\mathrm{HC}$ & $Q$ & Medium \\
\hline Perrin et $a l^{18}$ & $\mathrm{Y}$ & $\mathrm{N}$ & $\mathrm{Y}$ & $\mathrm{Y}$ & $\mathrm{Y}$ & CFS vs $\mathrm{HC}$ & $\mathrm{N}$ & High \\
\hline Greco et $a l^{19}$ & Y & Y & Y & Y & Y & CFS vs $\mathrm{HC}$ & $\mathrm{N}$ & Medium \\
\hline Schwartz et $\left.a\right|^{20}$ & $\mathrm{Y}$ & Y & Y & Y & Y & CFS vs HC & $\mathrm{N}$ & Medium \\
\hline Lange et $a l^{21}$ & Y & Y & Y & Y & Y & CFS vs $\mathrm{HC}$ & $\mathrm{N}$ & Medium \\
\hline Lange et $a l^{22}$ & Y & Y & Y & Y & Y & CFS vs HC & $\mathrm{N}$ & Medium \\
\hline Zeineh et $\left.a\right|^{37}$ & Y & Y & Y & Y & Y & CFS vs $\mathrm{HC}$ & Q & Low \\
\hline
\end{tabular}

CFS, chronic fatigue syndrome; $\mathrm{HC}$, healthy controls.

\section{GENERAL DISCUSSION}

\section{Structural MRI}

This is the first systematic review of sMRI studies in CFS/ME. While authors were optimistic about finding a biomarker, the findings were inconsistent between studies which could be due to differences in methodology, sample sizes and underlying disease heterogeneity. Differences in methodology include using visual

Table 4 Shows risk of bias assessment for functional MRI studies

\begin{tabular}{|c|c|c|c|c|c|c|c|}
\hline Authors & $\begin{array}{l}\text { Research } \\
\text { objectives }\end{array}$ & $\begin{array}{l}\text { Recruitment } \\
\text { procedure }\end{array}$ & Inclusion/exclusion & $\begin{array}{l}\text { Population } \\
\text { demographics }\end{array}$ & $\begin{array}{l}\text { Imaging } \\
\text { protocol }\end{array}$ & $\begin{array}{l}\text { Comparison } \\
\text { group }\end{array}$ & $\begin{array}{l}\text { Risk of } \\
\text { bias }\end{array}$ \\
\hline Gay et $a l^{23}$ & $\mathrm{Y}$ & $Y$ & $Y$ & $Y$ & $Y$ & CFS vs HC & Low \\
\hline Kim et $a l^{24}$ & $\mathrm{Y}$ & $\mathrm{Y}$ & $\mathrm{Y}$ & $\mathrm{Y}$ & Y & CFS vs $\mathrm{HC}$ & Low \\
\hline Wortinger et $a^{25}$ & Y & Y & $\mathrm{Y}$ & $Y$ & Y & CFS vs HC & Low \\
\hline Wortinger et $\left.a\right|^{26}$ & $\mathrm{Y}$ & $\mathrm{Y}$ & $\mathrm{Y}$ & $\mathrm{Y}$ & $\mathrm{Y}$ & CFS vs $\mathrm{HC}$ & Low \\
\hline Cook et $a l^{27}$ & Y & Y & $\mathrm{Y}$ & $\mathrm{Y}$ & Y & CFS vs $\mathrm{HC}$ & Low \\
\hline Mizuno et $\left.a\right|^{28}$ & $\mathrm{Y}$ & $\mathrm{Y}$ & $\mathrm{Y}$ & $\mathrm{Y}$ & $\mathrm{Y}$ & CFS vs $\mathrm{HC}$ & Low \\
\hline Mizuno et $\left.a\right|^{29}$ & $\mathrm{Y}$ & $\mathrm{Y}$ & $\mathrm{Y}$ & Y & Y & CFS vs $\mathrm{HC}$ & Low \\
\hline de Lange et $a^{30}$ & $\mathrm{Y}$ & $\mathrm{Y}$ & $\mathrm{Y}$ & $\mathrm{Y}$ & $\mathrm{Y}$ & CFS vs $\mathrm{HC}$ & Low \\
\hline Lange et $a^{31}$ & $\mathrm{Y}$ & $\mathrm{Y}$ & Y & Y & Y & CFS vs HC & Low \\
\hline Tanaka et $a^{32}$ & $\mathrm{Y}$ & $\mathrm{Y}$ & $\mathrm{Y}$ & $\mathrm{Y}$ & $\mathrm{Y}$ & CFS vs HC & Low \\
\hline Caseras et $\mathrm{a} /{ }^{33}$ & $\mathrm{Y}$ & $\mathrm{Y}$ & $\mathrm{Y}$ & Y & Y & CFS vs HC & Low \\
\hline Caseras et $a l^{34}$ & $\mathrm{Y}$ & $\mathrm{Y}$ & $\mathrm{Y}$ & $\mathrm{Y}$ & $\mathrm{Y}$ & CFS vs $\mathrm{HC}$ & Low \\
\hline Wortinger et $a \beta^{35}$ & Y & $\mathrm{Y}$ & $\mathrm{Y}$ & $\mathrm{Y}$ & Y & CFS vs $\mathrm{HC}$ & Low \\
\hline Miller et $a l^{42}$ & $\mathrm{Y}$ & $\mathrm{Y}$ & $\mathrm{Y}$ & $\mathrm{Y}$ & $\mathrm{Y}$ & CFS vs HC & Low \\
\hline Shan et $a{ }^{40}$ & Y & $Y$ & Y & Y & Y & CFS vs $\mathrm{HC}$ & Low \\
\hline Cook et a ${ }^{41}$ & $\mathrm{Y}$ & $\mathrm{Y}$ & $\mathrm{Y}$ & $\mathrm{Y}$ & $\mathrm{Y}$ & CFS vs HC & Low \\
\hline
\end{tabular}

CFS, chronic fatigue syndrome; HC, healthy controls. 
inspection, computational analysis, different sample sizes and patients with CFS/ME with different duration of illness or symptom severity. The lack of automated analysis methods showed inconsistencies and found no differences between CFS/ME and healthy control groups in studies that used visual inspection. ${ }^{17} 19212244$ Studies reporting white matter changes, such as white matter hyperintensities and ventricular enlargement are not specific to CFS/ME. Illnesses such as multiple sclerosis also have a similar pattern of white matter alterations. As a result, the use of the automated analysis method might be crucial in improving the ability to find differences between brain regions in these subgroups.

The results from quantitative studies using automated analysis of brain volume in the CFS/ME group compared with controls showed reductions in brain volume in midbrain, pons and right temporal lobe, ${ }^{13}$ left inferior fronto-occipital fasciculus ${ }^{8} 15$ and left occipital lobe ${ }^{14}$ while white matter abnormalities were reported in right middle temporal lobe. ${ }^{7}$ White matter volume reduction and abnormalities were found to be related to cognition. ${ }^{45}$ White matter facilitates information transfer in the brain to enable fast and effective neural systems which is essential for cognitive operations. ${ }^{45}$ Any disturbance in these neural networks would affect aspects of cognition such as memory, visuospatial skills, language, attention and executive function, which rely on structural connectivity delivered by the myelinated systems. ${ }^{45}$ Barnden et al concluded that although the brain regulatory nuclei are working, the signalling to and from the peripheral sensor might be affected due to impairment of the two-way communication. ${ }^{6}$ Shan et al found a reduction in inferior fronto-occipital fasciculus and an association with working memory deficits, impaired concentration, poor motor coordination and inability to focus vision. This region plays a role in connecting the frontal lobe with the superior parietal lobe. In addition, the ventral subcomponent of inferior fronto-occipital fasciculus connects the frontal lobe with the inferior occipital lobe and the temporobasal area. ${ }^{15} 46$

Five studies showed a reduction in grey matter volume. Three of these showed a global reduction ${ }^{5910}$ and two showed a reduction in specific regions of the brain (occipital lobes, right angular gyrus and the posterior division left parahippocampal gyrus ${ }^{14}$ and in bilateral prefrontal cortex $\left.{ }^{11}\right)$. Grey matter reduction is of particular interest because it provides a possible explanation for the memory problems seen in CFS/ME. Puri et al found a reduction in grey matter volume in the posterior part of the left parahippocampal gyrus ${ }^{14}$ which has been shown to be affected in other diseases like age-related memory decline. ${ }^{47}$ Reduced grey matter volume was found among the CFS/ME group and was suggested to be a reason for neuronal downregulation which might be caused by environmental impoverishment associated with the disease. ${ }^{10}$ In one study, neurogenesis was directly correlated with physical exercise. ${ }^{9}$ However, given the nature of these cross-sectional studies, further research is required to support this hypothesis.
Three studies investigated CFS/ME longitudinally with varying periods (6-9 months with CBT, 1 year and 6 years)..$^{10} 15$ sMRI showed evidence of CBT treatment effects on brain volume during a longitudinal study. ${ }^{10}$ Moreover, the increase in grey matter volume indicates macroscopic cortical plasticity in the human brain and suggests that there is a dynamic relationship between cerebral anatomy and behavioural state. ${ }^{10}$ Furthermore, the increase in grey matter volume after CBT might be agedependant as younger CFS/ME participants showed more improvement compared with older participants. ${ }^{10}$ Shan et al showed a reduction in left inferior fronto-occipital fasciculus (white matter) during a 6-year longitudinal study ${ }^{15}$ but this was not consistent with Perrin $e t$ al who conducted a 1-year follow-up. ${ }^{18}$ These differences in findings could be due to differences in follow-up time. CFS/ ME may be a slow progressing illness, which is supported by the fact that neurodysfunction is related to the duration of illness. ${ }^{57}$ Alternatively, it could be due to different populations studied, as none of the studies defined their CFS/ME population accounting for subgroups.

Pain is an important factor which can occur at multiple sites, from the cerebral cortex to the spinal cord and is believed to be caused by maladaptive functional or structural plasticity of the nociceptive system. ${ }^{48}$ Pain is a common symptom in CFS/ME but not a primary symptom for diagnosis. Altered brain morphology on sMRI has been reported in many types of pain disorders, including chronic back pain ${ }^{49-51}$ chronic tension-type headache, ${ }^{52}$ fibromyalgia, ${ }^{53}{ }^{54}$ migraine ${ }^{55-57}$ and somatoform pain disorder, ${ }^{58}$ thus, not unique to CFS/ME.

Moreover, sample sizes were usually small, less than 30 in most studies (12/19). CFS/ME is a heterogeneous condition, and therefore conflicting results could be caused by studying different phenotypes with different underlying disease mechanisms. ${ }^{59} \mathrm{CFS} / \mathrm{ME}$ does not currently have any biomarkers or clinical signs therefore diagnosis is based on self-reported symptoms and excluding alternative explanations for diagnosis. The use of self-reported symptoms leads to doubt about the validity of CFS/ME as an aetiologically homogeneous diagnosis. ${ }^{6061}$ This, in turn, has produced research to empirically define cases and subgroups examining the heterogeneity of CFS/ME. Hickie et al used symptoms and demographics to empirically define a core group and a smaller polysymptomatic subgroup. ${ }^{62}$ A more recent study by Williams et al used latent class analysis to empirically define subgroups in a sample of 541 patients with CFS/ME and found five subgroups. ${ }^{63}$ This indicates that CFS/ME populations being studied may not have similar disease phenotypes, potentially resulting in the inconsistent findings.

\section{Functional MRI}

Most fMRI studies reported differences between CFS/ME participants and healthy controls in brain activity despite a lack of differences being detected in cognitive performance. Studies that investigated CFS/ME using rs-fMRI observed reduced functional connectivity of the salience 
network, which was interpreted as an altered or immature resting state network. ${ }^{23} 242635$ In addition, investigating the default mode network showed a more complex and less coordinated network in the CFS/ME group. ${ }^{40}$ The authors suggested that brain network analysis could be a potential diagnostic biomarker for this disease. Kim et al hypothesised that this abnormal connectivity might be a result of a cognitive and emotional deficit in this group. ${ }^{24}$ The salience network plays a major role in the connection between other brain networks such as the detection and integration of salient sensory information ${ }^{64} 65$ and switching between default mode network and central executive network. ${ }^{66}$ The altered functional connectivity may cause a disruption in the integration of important information, specifically for cognition. ${ }^{67}$ Moreover, disorders such as Alzheimer's disease and multiple sclerosis, with attentional disruptions, have been associated with the presence of abnormalities of the default mode network. These diseases overlap with CFS/ME in clinical features such as attention and memory difficulties. ${ }^{23} \mathrm{Kim}$ et $a l$ argued that the presence of default mode network deficit could be metabolically expensive thereby contributing to, or a causal factor of, fatigue, cognitive symptoms and postexertional malaise of CFS/ME. ${ }^{24}$

Differences in task difficulty may play a major role in why some studies reported increased activation while others reported decreased activation. Bryer et al conducted a meta-analysis of fMRI studies of memory function in patients with traumatic brain injury and concluded that the primary reason for the discrepancy in activation patterns across studies is attributable to task classification, where hyperactivation may be associated with continuous memory tasks and hypoactivation may be more prominent in discrete memory tasks. ${ }^{68}$ There have been a wide variety of tasks used in fMRI studies to assess differences between participants with CFS/ME and healthy controls. When rs-fMRI or simple tasks were employed, participants with CFS/ME showed decreased functional connectivity in various brain regions. ${ }^{23-28} 354042$ However, when more challenging tasks are employed, participants with CFS/ME exhibit widespread increased activation in task-related regions when compared with healthy controls. ${ }^{27} 2931333441$ Most of the CFS/ME participants performed at a similar level to healthy controls and it is not clear whether the increased activation was due to the increase in the task difficulty or because CFS/ ME participants were trying harder. This widespread activation may lead to an increase in demand on neural resources such as oxygen and glucose which in turn would lead to fatigue. ${ }^{10}$ Fatigue and lower performance have been associated with increased brain activity while performing a high-effort cognitive task. ${ }^{9}{ }^{27} 29$ It has been hypothesised that severe fatigue consumes a significant amount of attentional resources in terms of recruiting additional brain regions for cognitive compensation to perform better in dual task depending on the degree of mental effort. ${ }^{29}{ }^{33}$ Caseras $e t$ al suggested that the fear of being fatigued leads the CFS/ME group to avoid activity. ${ }^{33}$
Impaired reward processing was suggested to decrease motivation to learn in adolescents with $\mathrm{CFS} / \mathrm{ME},{ }^{28}$ while participants' inability to engage the part of the brain (left amygdala and left midposterior insula) that responds to conflict suggested an abnormal salience network functioning in terms of effect and cognition. ${ }^{25}$ The increased activity in task-related areas was hypothesised to be a result of cognitive and emotional deficits in participants with $\mathrm{CFS} / \mathrm{ME}^{24} 33$ and impaired reward processing in adolescents. ${ }^{29}$ Participants with CFS/ME failed to recruit working memory regions to the same level as the healthy controls, as evidenced by reduced activation when the task difficulty increased. ${ }^{33}$

The heterogeneity of tasks, behaviours and cognitive processes across the fMRI studies makes it difficult to discern how much of the increase or decrease in activation, reported in relation to task difficulty or demand, is associated with increasing cognitive fatigue. Understanding the impact of fatigue on brain function will be critical to our understanding of CFS/ME.

\section{LIMITATIONS}

Our systematic review has highlighted a limitation of fMRI studies in CFS/ME, which is the small sample sizes. Empirical and simulation studies conducted by Desmond and Glover $^{69}$ found that to achieve $80 \%$ power at the single voxel level for typical activations in fMRI studies with thresholds correcting for multiple comparisons a sample size of 24 is required. We found that 15 of the 16 fMRI studies had a patient sample size of less than 24 . Studies with low power reduce the likelihood of detecting a true effect, increase the risk of false negatives and the likelihood of false positives by reducing the positive predictive value of the test. However, this is not unique to neuroimaging studies in CFS/ME. fMRI studies have been criticised for being underpowered due to small sample sizes resulting in overestimates of effect size and low reproducibility. ${ }^{7071}$

All the studies in this systematic review did not report the signal to noise ratio (SNR) in their MRI methods. The SNR compares the level of the signal of interest to the level of background noise. In MRI studies SNR is important for comparison between different MRI scanners, imaging protocols and MR sequences. ${ }^{72}$ Thus, this limits this systematic review's ability to do a comprehensive comparative analysis.

The automated computational methods for investigating structural anatomical differences may be superior to subjective visual inspection but does have some limitations. Voxel-based morphometric analysis has been criticised for being significantly biased towards group differences that are highly localised in space and of a linear nature. In addition, these techniques are poor at detecting group differences that are spatially complex and subtle. ${ }^{73}$ The fMRI studies reported both increases and decreases in activation patterns in CFS/ME compared with controls; while this may be related to task demands, 
caution must also be taken when interpreting these results, bearing in mind fMRI is an indirect measure of neural activity. The fMRI signal is derived from the BOLD contrast mechanism, that is, haemodynamics of the brain. Currently, we cannot easily estimate the cerebral metabolic rate of oxygen from the BOLD signal. Furthermore, haemodynamic responses are sensitive to the size of the activated population, and less likely to detect cortical regions in which stimulus- or task-related perceptual or cognitive capacities have sparse neuronal representation. It is also not fully understood how neuromodulation might contribute to the spatiotemporal resolution of the fMRI signal. ${ }^{74}$ In recent years there has been a shift placing a greater emphasis on neural networks underlying behaviour and cognition. A functional connectivity approach considering the neural network difference between patients and healthy populations may lead to a better understanding of how the disease affects brain function.

The main limitation of the present systematic review is that there were insufficient data for meta-analysis. Metaanalysis of neuroimaging data can take two approaches, image-based analysis or coordinate-based analysis. Imagebased analysis requires the statistical images of the data and this is not often available due to data sharing issues, for example, data protection and other restrictions. Therefore, most neuroimaging meta-analyses are coordinatebased as these are reported in the published research. Moreover, the spatial normalisation of images into standardised coordinates as anatomical addresses within a reference space has been applied to human neuroimaging data for decades. ${ }^{756}$ In order to perform an appropriate coordinate-based meta-analysis some minimum criteria need to be met. First is power of the meta-analysis. For coordinate-based meta-analysis the activation likelihood estimation (ALE) method is conventionally applied, ${ }^{77}$ or a revised ALE algorithm. ${ }^{78}$ Based on a recent simulation study performed by Eickhoff $e t a l$, a recommendation was made to include at least 17-20 experiments in ALE metaanalyses for sufficient power to detect smaller effects and ensure results are not driven by single experiments. ${ }^{79}$ For sMRI we have 19 studies; 6 of these are visual inspection thus there is subjective reporting without quantitative coordinate data. Of the 13 quantitative studies only 6 studies reported coordinates. A meta-analysis of six sMRI studies would be severely underpowered.

For fMRI we have 16 studies in total, 5 fMRI studies are rs-fMRI and 11 task-based fMRI. The methodological differences between them preclude us from combining the results to perform a coordinate-based meta-analysis as they did not all meet the two minimum criteria. First, all studies must be whole brain analyses and second, they must use the same standardised coordinate system. Four rs-fMRI use Regions of Interest (ROI) seed regions and one uses the whole brain approach for calculating connectivity. For task-based fMRI studies, the task selection criteria are critical. In our systematic review we identified 11 task-based fMRI studies. However only two studies use the same PASAT task. The remaining nine studies all use different tasks and more importantly each task is designed to examine a different cognitive, sensory or physical function. Therefore, the heterogeneity of taskbased fMRI studies prohibits the creation of task selection criteria for meta-analysis.

\section{FUTURE DIRECTIONS}

The complexity of this illness and its related symptoms as well as using small sample sizes without controlling for population heterogeneity may explain the inconsistencies found in the literature. Future studies should use larger sample sizes with subgrouping according to the phenotypes and classification of participants according to CFS/ ME severity and symptom patterns. Subphenotyping ${ }^{59}$ could reduce the heterogeneity of the patient samples in case of control studies. Stratifying by symptoms, activity or sleep patterns may enable researchers to compare CFS/ ME to other conditions (or healthy controls). Only one research group has matched controls for sleep pattern, ${ }^{8}$ which is altered in CFS/ME, and is known to have a strong association with the BOLD signal measured by fMRI, grey matter and white matter volumes, ${ }^{80-83}$ suggesting that better matching between participants with CFS/ME and control group is required.

An additional important aspect is the use of longitudinal MRI data. Longitudinal studies enable us to examine the progression of structural changes in CFS/ ME, while controlling for age-related effect points. ${ }^{1015} 18$ In this systematic review, out of the 13 quantitative studies, 11 corrected for age in their statistical analysis. It is also important to measure whether the use of a treatment method is effective. The findings from longitudinal studies ${ }^{1015}$ demonstrate the importance of using two time points to understand the impact that treatment, length of illness or symptom severity may have on MRI volumetric measures.

Brain white matter volume increases linearly with age in adolescence ${ }^{84}$ and given the prevalence of CFS/ME in this age group ${ }^{85}$ more research is required to determine if there are distinct neurobiological markers comparable to studies in adults with CFS/ME. For sMRI studies, as quantitative automated methods found differences, ${ }^{5-11}$ 13-15 37 future research should focus on using automated objective methods. By using voxel-based morphometry, de Lange $e t$ al were able to show that improvement after CBT was associated with improving grey matter volume in the CFS/ME group. ${ }^{10}$ Shan et al were able to find progressive brain changes after a 6-year follow-up which therefore led us to conclude that sMRI studies might not yet show evidence as a diagnostic tool, but can be used as an objective measure of treatment evaluation. Consistency in research might be achieved by using standardised MRI protocols which have been evaluated and compared with other illnesses such as the use of standard MRI protocols across multiple sites in Alzheimer's disease. ${ }^{86}$ Neuroimaging researchers can use the views of CFS/ME experts 
regarding different grouping strategies which can aid in finding CFS/ME biomarkers which may steer CFS/ME research in directions that hold promise and eventually help clinicians in the optimisation of their practices.

Finally, we have limited this systematic review to include only neuroimaging studies that have used sMRI or fMRI methods. However, other neuroimaging techniques have been used to investigate CFS/ME; these include single-photon emission CT, electroencephalogram, MR spectroscopy and diffusion tensor imaging. These methods measure different neurophysiologies from sMRI and fMRI. Future studies using multimodel imaging approaches could overcome some of the limitations of a single method alone.

\section{CONCLUSION}

In conclusion, there is no evidence to support the assertion that findings from neuroimaging studies have found any clear biomarkers of CSF/ME. However, MRI can be considered a powerful tool if rigorous procedures for the collection of data and analysis are employed, taking into account the limitations of the neurophysiology being measured by fMRI. There is a significant need for more research, given the sparsity of studies, as is evident by our inability to conduct a meta-analysis. MRI studies in this systematic review have demonstrated the potential for significant insights into CFS/ME which is not afforded by other techniques. For example, fMRI studies have provided objective measures of the impact of fatigue experienced by participants with CFS/ME on cognition, even in the absence of behavioural and cognitive deficits. $^{2731}$ sMRI has shown evidence of treatment effects on brain volume ${ }^{10}$ while fMRI has demonstrated functional connectivity changes and altered patterns of activation. Future MRI studies could potentially, with proper study design, subgrouping and sample size, lead to a breakthrough in our understanding of this illness.

Acknowledgements The authors thank Ms Catherine Borwick, University of Bristol librarian, for her help in the search stage of this review.

Contributors BA created systematic review protocol, conducted online literature search, reviewed and selected articles for inclusion, conducted full-text review of all articles, data extraction, synthesis and quality assessment, and wrote this manuscript. CL reviewed search articles, read full texts of articles for inclusion and edited this manuscript. EC conceived the systematic review topic, and reviewed and edited the protocol. She also reviewed and edited this manuscript. NJT reviewed and edited the protocol, read full-text articles included in the systematic review for quality assessment, reviewed and edited data extraction and synthesis. and reviewed and edited the manuscript for submission.

Funding BA is supported by the Saudi Ministry of Health PhD Scholarship at the University of Bristol.

Competing interests None declared.

Patient consent for publication Not required.

Provenance and peer review Not commissioned; externally peer reviewed.

Data availability statement All data relevant to the study are included in the article or uploaded as supplementary information.

Open access This is an open access article distributed in accordance with the Creative Commons Attribution Non Commercial (CC BY-NC 4.0) license, which permits others to distribute, remix, adapt, build upon this work non-commercially, and license their derivative works on different terms, provided the original work is properly cited, appropriate credit is given, any changes made indicated, and the use is non-commercial. See: http://creativecommons.org/licenses/by-nc/4.0/.

ORCID iD

Ngoc Jade Thai http://orcid.org/0000-0001-6125-7326

\section{REFERENCES}

1 Collin SM, Nikolaus S, Heron J, et al. Chronic fatigue syndrome (CFS) symptom-based phenotypes in two clinical cohorts of adult patients in the UK and the Netherlands. J Psychosom Res 2016;81:14-23.

2 Johnston S, Brenu EW, Staines D, S. Johnston, E.W.B, MarshallGradisnik S, et al. The prevalence of chronic fatigue syndrome/ myalgic encephalomyelitis: a meta-analysis. Clin Epidemiol 2013;5:105.

3 Collin SM, Crawley E, May MT, et al. The impact of CFS/ME on employment and productivity in the UK: a cross-sectional study based on the CFS/ME national outcomes database. BMC Health Serv Res 2011;11:217.

4 Nichols TE, Das S, Eickhoff SB, et al. Best practices in data analysis and sharing in neuroimaging using MRI. Nat Neurosci 2017;20:299-303.

5 Barnden LR, Crouch B, Kwiatek R, et al. A brain MRI study of chronic fatigue syndrome: evidence of brainstem dysfunction and altered homeostasis. NMR Biomed 2011;24:1302-12.

6 Barnden LR, Kwiatek R, Crouch B, et al. Autonomic correlations with MRI are abnormal in the brainstem vasomotor centre in chronic fatigue syndrome. Neuroimage Clin 2016;11:530-7.

7 Barnden LR, Crouch B, Kwiatek R, Benjamin C, Richard K, et al. Evidence in chronic fatigue syndrome for severity-dependent upregulation of prefrontal myelination that is independent of anxiety and depression. NMR Biomed 2015;28:404-13.

8 Shan ZY, Kwiatek R, Burnet R, et al. Medial prefrontal cortex deficits correlate with unrefreshing sleep in patients with chronic fatigue syndrome. NMR Biomed 2017;30:nbm.3757.

9 de Lange FP, Kalkman JS, Bleijenberg G, et al. Gray matter volume reduction in the chronic fatigue syndrome. Neuroimage 2005;26:777-81.

10 de Lange FP, Koers A, Kalkman JS, et al. Increase in prefrontal cortical volume following cognitive behavioural therapy in patients with chronic fatigue syndrome. Brain 2008;131:2172-80.

11 Okada T, Tanaka M, Kuratsune $\mathrm{H}$, et al. Mechanisms underlying fatigue: a voxel-based morphometric study of chronic fatigue syndrome. BMC Neurol 2004;4:14.

12 van der Schaaf ME, De Lange FP, Schmits IC, et al. Prefrontal structure varies as a function of pain symptoms in chronic fatigue syndrome. Biol Psychiatry 2017;81:358-65.

13 Finkelmeyer A, He J, Maclachlan L, et al. Grey and white matter differences in Chronic Fatigue Syndrome - A voxel-based morphometry study. Neuroimage Clin 2018;17:24-30.

14 Puri BK, Jakeman PM, Agour M, et al. Regional grey and white matter volumetric changes in myalgic encephalomyelitis (chronic fatigue syndrome): a voxel-based morphometry $3 \mathrm{~T}$ MRI study. $\mathrm{Br} \mathrm{J}$ Radiol 2012;85:e270-3.

15 Shan ZY, Kwiatek R, Burnet R, et al. Progressive brain changes in patients with chronic fatigue syndrome: a longitudinal MRI study. $J$ Magn Reson Imaging 2016;44:1301-11.

16 Barnden LR, Shan ZY, Staines DR, et al. Hyperintense sensorimotor T1 spin echo MRI is associated with brainstem abnormality in chronic fatigue syndrome. Neuroimage 2018;20:102-9.

17 Natelson BH, Cohen JM, Brassloff I, et al. A controlled study of brain magnetic resonance imaging in patients with the chronic fatigue syndrome. J Neurol Sci 1993;120:213-7.

18 Perrin R, Embleton K, Pentreath VW, et al. Longitudinal MRI shows no cerebral abnormality in chronic fatigue syndrome. $\mathrm{Br} J$ Radiol 2010;83:419-23.

19 Greco A, Tannock C, Brostoff J, et al. Brain Mr in chronic fatigue syndrome. AJNR Am J Neuroradiol 1997;18:1265-9.

20 Schwartz RB, Garada BM, Komaroff AL, et al. Detection of intracranial abnormalities in patients with chronic fatigue syndrome: comparison of Mr imaging and SPECT. American Journal of Roentgenology 1994;162:935-41.

21 Lange G, DeLuca J, Maldjian JA, et al. Brain MRI abnormalities exist in a subset of patients with chronic fatigue syndrome. J Neurol Sci 1999;171:3-7.

22 Lange G, Holodny Al, DeLuca J, et al. Quantitative assessment of cerebral ventricular volumes in chronic fatigue syndrome. Appl Neuropsychol 2001;8:23-30. 
23 Gay CW, Robinson ME, Lai S, et al. Abnormal resting-state functional connectivity in patients with chronic fatigue syndrome: results of seed and data-driven analyses. Brain Connect 2016;6:48-56.

24 Kim B-H, Namkoong K, Kim J-J, et al. Altered resting-state functional connectivity in women with chronic fatigue syndrome. Psychiatry Res 2015;234:292-7.

25 Wortinger LA, Endestad T, Melinder AMD, et al. Emotional conflict processing in adolescent chronic fatigue syndrome: a pilot study using functional magnetic resonance imaging. J Clin Exp Neuropsychol 2017;39:355-68.

26 Wortinger LA, Glenne Øie M, Endestad T, et al. Altered right anterior insular connectivity and loss of associated functions in adolescent chronic fatigue syndrome. PLoS One 2017;12:e0184325.

27 Cook DB, O'Connor PJ, Lange G, et al. Functional neuroimaging correlates of mental fatigue induced by cognition among chronic fatigue syndrome patients and controls. Neuroimage 2007;36:108-22.

28 Mizuno K, Kawatani J, Tajima K, et al. Low putamen activity associated with poor reward sensitivity in childhood chronic fatigue syndrome. Neuroimage Clin 2016;12:600-6.

29 Mizuno K, Tanaka M, Tanabe HC, et al. Less efficient and costly processes of frontal cortex in childhood chronic fatigue syndrome. Neuroimage Clin 2015;9:355-68.

30 de Lange FP, Kalkman JS, Bleijenberg G, et al. Neural correlates of the chronic fatigue syndrome--an fMRI study. Brain 2004;127:1948-57.

31 Lange G, Steffener J, Cook DB, et al. Objective evidence of cognitive complaints in chronic fatigue syndrome: a BOLD fMRI study of verbal working memory. Neuroimage 2005;26:513-24.

32 Tanaka M, Sadato N, Okada T, et al. Reduced responsiveness is an essential feature of chronic fatigue syndrome: a fMRI study. BMC Neurol 2006;6:9.

33 Caseras X, Mataix-Cols D, Giampietro V, et al. Probing the working memory system in chronic fatigue syndrome: a functional magnetic resonance imaging study using the n-back task. Psychosom Med 2006;68:947-55.

34 Caseras X, Mataix-Cols D, Rimes KA, et al. The neural correlates of fatigue: an exploratory imaginal fatigue provocation study in chronic fatigue syndrome. Psychol Med 2008;38:941-51.

35 Wortinger LA, Endestad T, Melinder AMD, et al. Aberrant restingstate functional connectivity in the salience network of adolescent chronic fatigue syndrome. PLoS One 2016;11:e0159351.

36 Scheltens $P$, Barkhof $F$, Leys $D$, et al. A semiquantative rating scale for the assessment of signal hyperintensities on magnetic resonance imaging. J Neurol Sci 1993:114:7-12.

37 Zeineh MM, Kang J, Atlas SW, et al. Right arcuate fasciculus abnormality in chronic fatigue syndrome. Radiology 2015;274:517-26.

38 Geha PY, Baliki MN, Harden RN, et al. The brain in chronic CRPS pain: abnormal gray-white matter interactions in emotional and autonomic regions. Neuron 2008;60:570-81.

39 Kuchinad A, Schweinhardt P, Seminowicz DA, et al. Accelerated brain gray matter loss in fibromyalgia patients: premature aging of the brain? J Neurosci 2007;27:4004-7.

40 Shan ZY, Finegan K, Bhuta S, et al. Decreased connectivity and increased blood oxygenation level dependent complexity in the default mode network in individuals with chronic fatigue syndrome. Brain Connect 2018;8:33-9.

41 Cook DB, Light AR, Light KC, et al. Neural consequences of postexertion malaise in myalgic Encephalomyelitis/Chronic fatigue syndrome. Brain Behav Immun 2017;62:87-99.

42 Miller AH, Jones JF, Drake DF, et al. Decreased basal ganglia activation in subjects with chronic fatigue syndrome: association with symptoms of fatigue. PLoS One 2014;9:e98156.

43 Menon V. Salience network. In: Toga AW, ed. Brain mapping: an encyclopedic reference. London: Academic Press, 2015: 2. 597-611.

44 Schwartz R, Garada BM, Komaroff AL, et al. Detection of Intracracial Abnormalities in Patients with Chronic Fatigue Syndrome: Comparison of MR Imaging and SPECT, 1993.

45 Filley $\mathrm{CM}$, Fields RD. White matter and cognition: making the connection. J Neurophysiol 2016;116:2093-104.

46 Martino J, Brogna C, Robles SG, et al. Anatomic dissection of the inferior fronto-occipital fasciculus revisited in the lights of brain stimulation data. Cortex 2010;46:691-9.

47 Burgmans S, van Boxtel MPJ, van den Berg KEM, v.B.M.P. vandenBKE, Gronenschild EH, et al. The posterior parahippocampal gyrus is preferentially affected in age-related memory decline. Neurobiol Aging 2011;32:1572-8.

48 Woolf CJ, Salter MW. Neuronal plasticity: increasing the gain in pain. Science 2000;288:1765-8.
49 Apkarian AV, Sosa Y, Sonty S, et al. Chronic back pain is associated with decreased prefrontal and thalamic gray matter density. $J$ Neurosci 2004;24:10410-5.

50 Buckalew N, Haut MW, Morrow L, et al. Chronic pain is associated with brain volume loss in older adults: preliminary evidence. Pain Med 2008;9:240-8

51 Schmidt-Wilcke T, Leinisch E, Gänssbauer S, et al. Affective components and intensity of pain correlate with structural differences in gray matter in chronic back pain patients. Pain 2006;125:89-97.

52 Schmidt-Wilcke T, Leinisch E, Straube A, et al. Gray matter decrease in patients with chronic tension type headache. Neurology 2005;65:1483-6.

53 Hsu MC, Harris RE, Sundgren PC, et al. No consistent difference in gray matter volume between individuals with fibromyalgia and age-matched healthy subjects when controlling for affective disorder. Pain 2009;143:262-7.

54 Burgmer M, Gaubitz M, Konrad C, et al. Decreased gray matter volumes in the cingulo-frontal cortex and the amygdala in patients with fibromyalgia. Psychosom Med 2009;71:566-73.

$55 \mathrm{Kim} \mathrm{JH}$, Suh S-I, Seol HY, et al. Regional grey matter changes in patients with migraine: a voxel-based morphometry study. Cephalalgia 2008;28:598-604.

56 Schmidt-Wilcke T, Gänssbauer S, Neuner T, et al. Subtle grey matter changes between migraine patients and healthy controls. Cephalalgia 2008;28:1-4.

57 Valfrè W, Rainero I, Bergui M, et al. Voxel-Based morphometry reveals gray matter abnormalities in migraine. Headache 2008:48:109-17.

58 Valet M, Gündel H, Sprenger T, et al. Patients with pain disorder show gray-matter loss in pain-processing structures: a voxel-based morphometric study. Psychosom Med 2009;71:49-56.

59 Hickie I, Davenport T, Vernon, R SD, et al. International chronic fatigue syndrome study, are chronic fatigue and chronic fatigue syndrome valid clinical entities across countries and health-care settings? Aust N Z J Psychiatry 2009;43:25-35.

60 Komaroff AL. Myalgic Encephalomyelitis/Chronic fatigue syndrome: a real illness. Ann Intern Med 2015;162:871-2.

61 Wakefield JC. The DSM-5 debate over the bereavement exclusion: psychiatric diagnosis and the future of empirically supported treatment. Clin Psychol Rev 2013;33:825-45.

62 Hickie I, Lloyd A, Hadzi-Pavlovic D, et al. Can the chronic fatigue syndrome be defined by distinct clinical features? Psychol Med 1995;25:925-35.

63 Williams TE, Chalder T, Sharpe M, et al. Heterogeneity in chronic fatigue syndrome - empirically defined subgroups from the PACE trial. Psychol Med 2017;47:1454-65.

64 Seeley WW, Menon V, Schatzberg AF, et al. Dissociable intrinsic connectivity networks for salience processing and executive control. $J$ Neurosci 2007;27:2349-56.

65 Chen T, Michels L, Supekar K, et al. Role of the anterior insular cortex in integrative causal signaling during multisensory auditoryvisual attention. Eur J Neurosci 2015;41:264-74.

66 Sridharan D, Levitin DJ, Menon V. A critical role for the right frontoinsular cortex in switching between central-executive and defaultmode networks. Proc Natl Acad Sci U S A 2008;105:12569-74.

67 Toga AW. Brain mapping. In: An encyclopedic reference, 2015.

68 Bryer EJ, Medaglia JD, Rostami S, et al. Neural recruitment after mild traumatic brain injury is task dependent: a meta-analysis. $J$ Int Neuropsychol Soc 2013;19:751-62.

69 Desmond JE, Glover GH. Estimating sample size in functional MRI (fMRI) neuroimaging studies: statistical power analyses. J Neurosci Methods 2002;118:115-28.

70 Button KS, loannidis JPA, Mokrysz C, et al. Power failure: why small sample size undermines the reliability of neuroscience. Nat Rev Neurosci 2013:14:365-76.

71 Turner BO, Paul EJ, Miller MB, et al. Small sample sizes reduce the replicability of task-based fMRI studies. Commun Biol 2018:1

72 Welvaert M, Rosseel Y. On the definition of signal-to-noise ratio and contrast-to-noise ratio for fMRI data. PLoS One 2013;8:e77089.

73 Davatzikos C. Why voxel-based morphometric analysis should be used with great caution when characterizing group differences. Neuroimage 2004;23:17-20.

74 Miller EK, Cohen JD, Logothetis NK, et al. What we can do and what we cannot do with fMRI. Nature Neuroscience 2008:24:869-78.

75 Radua J, Mataix-Cols D. Meta-Analytic methods for neuroimaging data explained. Biol Mood Anxiety Disord 2012;2:6.

76 Müller VI, Cieslik EC, Laird AR, et al. Ten simple rules for neuroimaging meta-analysis. Neurosci Biobehav Rev 2018:84:151-61. 
77 Turkeltaub PE, Eden GF, Jones KM, et al. Meta-Analysis of the functional neuroanatomy of single-word reading: method and validation. Neuroimage 2002;16:765-80.

78 Eickhoff SB, Laird AR, Grefkes C, et al. Coordinate-based activation likelihood estimation meta-analysis of neuroimaging data: a randomeffects approach based on empirical estimates of spatial uncertainty. Hum Brain Mapp 2009;30:2907-26.

79 Eickhoff SB, Nichols TE, Laird AR, et al. Behavior, sensitivity, and power of activation likelihood estimation characterized by massive empirical simulation. Neuroimage 2016;137:70-85.

80 Sexton CE, Betts JF, Demnitz N, et al. A systematic review of MRI studies examining the relationship between physical fitness and activity and the white matter of the ageing brain. Neuroimage 2016;131:81-90.
81 Burzynska AZ, Wong CN, Voss MW, et al. Physical activity is linked to greater Moment-To-Moment variability in spontaneous brain activity in older adults. PLoS One 2015;10:e0134819.

82 Erickson KI, Leckie RL, Weinstein AM. Physical activity, fitness, and gray matter volume. Neurobiol Aging 2014;35 Suppl 2:S20-8.

83 Krause AJ, Simon EB, Mander BA, et al. The sleep-deprived human brain. Nat Rev Neurosci 2017:18:404-18.

84 Giedd JN, Blumenthal J, Jeffries NO, et al. Brain development during childhood and adolescence: a longitudinal MRI study. Nat Neurosci 1999;2:861-3.

85 Collin SM, Norris T, Nuevo R, et al. Chronic fatigue syndrome at age 16 years. Pediatrics 2016;137:e20153434.

86 Jack CR, Bernstein MA, Fox NC, et al. The Alzheimer's disease neuroimaging Initiative (ADNI): MRI methods. J Magn Reson Imaging 2008;27:685-91. 